\title{
Features of tensoresistance in single crystals of germanium and silicon with different dopants
}

\author{
P.I. Baranskii ${ }^{1}$, G.P. Gaidar ${ }^{2}$ \\ ${ }^{I}$ V. Lashkaryov Institute of Semiconductor Physics, National Academy of Sciences of Ukraine, \\ 45, prospect Nauky, 03680 Kyiv, Ukraine \\ ${ }^{2}$ Institute for Nuclear Research, National Academy of Sciences of Ukraine, \\ 47, prospect Nauky, 03680 Kyiv, Ukraine; e-mail: gaydar@kinr.kiev.ua
}

\begin{abstract}
Tensoresistance in single crystals of germanium and silicon with different dopants but under practically equal charge carrier concentrations have been investigated. The features of $\rho_{X} / \rho_{0}=f(X)$ function, which depend on individual physical-chemical properties of dopants, have been discussed in this paper.
\end{abstract}

Keywords: germanium, silicon, dopant impurity, tensoresistance, anisotropy parameter of mobility.

Manuscript received 12.10.15; revised version received 18.01.16; accepted for publication 16.03.16; published online 08.04.16.

\section{Introduction}

Electronic properties of many-valley semiconductors are determined by their band structure as well as availability, energy spectrum and spatial distribution of electrically active impurities and defects in the bulk of crystal [1]. With mass production of semiconductor electronics devices, to meet the modern requirements of reproducibility and stability of their characteristics is possible only when using materials with high homogeneity of parameters [2]. This is particularly important in microelectronics in the manufacture of large- and very large-scale integrated microcircuits and power semiconductor devices. Today, it became clear that further increasing the density of integrated microcircuits, their reliability and efficiency, obviously, can not be realized only by improving technology and restricting microinhomogeneities of physicochemical properties in semiconductor materials [3]. Therefore, the problem of microinhomogeneities research has been and remains urgent to date [4].
A characteristic feature of $\mathrm{Ge}$ and $\mathrm{Si}$, as well as other semiconductor materials, is their high sensitivity to all kinds of perturbations in the periodicity of crystal lattice - crystal defects [5-7].

Defects are usually separated by two large groups: 1) one-dimensional, two-dimensional, three-dimensional ones that disturb the crystal structure at considerable distances; 2) point defects that disturb the crystal structure over relatively small distances. The first group includes dislocations, dislocation networks, grain boundaries and twins, different defects of packing, nuclei of a new phase, clusters of point defects. These defects are formed, as a rule, in the process of growing crystals, during plastic deformation, as well as under irradiation by heavy particles of a high energy.

Point defects, in turn, can be separated by two types: 1) the intrinsic point defects of lattice, and 2) the impurity atoms. Vacancy and interstitial atom belong to the simplest intrinsic point defects of the lattice.

Impurity atoms are the second major type of point defects, which are introduced into the semiconductor 
crystal during its growth, as well as under diffusion or by ion implantation. These point defects can be placed both in the lattice sites, and in the interstitial positions of the crystal lattice. If the impurity atom in its physical and chemical properties is similar to the atoms of matrix, then it is usually placed in a vacant lattice site. In the case when its properties are very different from those of matrix atoms, it is located between the lattice sites. In many-valley semiconductors, all atoms of the periodic system can serve as impurities. This fact allows obtaining a wide range of electronic properties of crystals $[8,9]$.

Some distortion of the interatomic bonds and the change of the lattice parameter occur at any position of the impurity atom, i.e., a defect in the crystal lattice appear. Character of these distortions depends both on the physical and chemical nature of the impurity atom as well as on the difference between the covalent radii of the atoms of impurity and matrix.

When the concentration of dopant impurity rises, interaction between impurity atoms appears and their energy levels are changed just as the atomic electronic levels split in the band during formation of the crystal. The impurity band appears as a result of this interaction. When the concentration of the impurity atoms with shallow levels is higher than $10^{18} \mathrm{~cm}^{-3}$, their ionization energy is reduced to almost zero as a result of the expansion of the impurity band and its confluence with the conduction or valence bands. Semiconductor acquires metallic properties; it becomes a semimetal or a degenerate semiconductor.

Dopant impurities affect on the character of charge carriers movement, and the latter are scattered by the ionized and neutral impurity atoms, which significantly affects on the phenomena of electronic transport [5]. Impurity atoms, as a lattice defects, affect on the other physical, chemical, optical, and magnetic properties of semiconductors, as well as actively interact with the radiation defects [10-12]. These processes lead to the change in the energy spectrum of the impurity states in the forbidden band.

Characteristic features of the physical properties of many-valley semiconductors are determined by the symmetry of crystal lattice and the nature of interatomic interaction. To investigate crystals, uniaxial elastic deformation is often applied, which causes the change not only in interatomic distances but also in symmetry of lattice, and results in the most significant changes in their energy spectrum [13]. This, in turn, determines the corresponding changes in their electronic properties, the study of which provides the valuable information about the investigated object [14].

The aim of this study was to determine the features of changes in resistivity under the influence of directed elastic deformation of germanium and silicon single crystals, which had about the same level of doping, but doped with different impurities, in particular, Ge crystals doped with antimony, silicon and arsenic and Si crystals doped with antimony, phosphorus and arsenic were used.

\section{Results and discussion}

By studying changes in the resistivity of the crystals under the influence of the directed elastic deformation $X$ and bringing the function $\rho=\rho(X)$ to saturation $\left(\rho_{\infty} / \rho_{0}\right)$, one can get the value of the anisotropy parameter of mobility $K$ in a single isoenergetic ellipsoid by using the following equation [15]:

$$
K=\frac{\mu_{\perp}}{\mu_{\|}}=\frac{3}{2} \frac{\rho_{\infty}^{\langle i j k\rangle}}{\rho_{0}}-\frac{1}{2},
$$

where $\mu_{\|}, \mu_{\perp}$ are the charge carrier mobilities along and across the long axis of the isoenergetic ellipsoid, respectively; $\rho_{0}, \rho_{\infty}-$ resistivities of unstrained $\rho(X=0) \equiv \rho_{0}$ and uniaxially elastically deformed crystals $\rho(X \rightarrow \infty) \equiv \rho_{\infty}$, which corresponds to the saturation region of $\rho=\rho(X)$, crystals in the crystallographic direction $\langle i j k\rangle$ (i.e., under condition $\vec{X}\|\vec{J}\|\langle i j k\rangle$, where $X$ is the mechanical load, $J-$ current); and $\langle i j k\rangle \rightarrow\left\{\begin{array}{l}\langle 111\rangle-\text { for } n-\mathrm{Ge}, \\ \langle 100\rangle-\text { for } n-\mathrm{Si} .\end{array}\right.$

To study the influence of the specific impurity on the tensoresistance and anisotropy parameter of mobility, the Ge single crystals were selected. These crystals have similar doping level $\left(\rho_{77.4 \mathrm{~K}} \approx\right.$ 0.8..0.9 Ohm.cm), but doped with As, $\mathrm{Si}, \mathrm{Sb}$, which atoms have significantly different tetrahedral radii $\left(r_{\mathrm{As}}=\right.$ $0.96 \AA, r_{\mathrm{Si}}=1.17 \AA, r_{\mathrm{Sb}}=1.36 \AA$ ). For example, let us consider Ge crystals $\left(r_{\mathrm{Ge}}=1.22 \AA\right)$ uniaxially elastically deformed in the crystallographic direction [111] and doped with impurities As and Sb, which tetrahedral radii up to 1.42 times differ. Fig. 1 shows that the dependence of tensoresistance $\rho_{X} / \rho_{0}$ on the mechanical stress $X$ for the samples $\mathrm{Ge}\langle\mathrm{As}\rangle$ are located significantly higher than the similar dependence for the samples $\mathrm{Ge}\langle\mathrm{Sb}\rangle$.

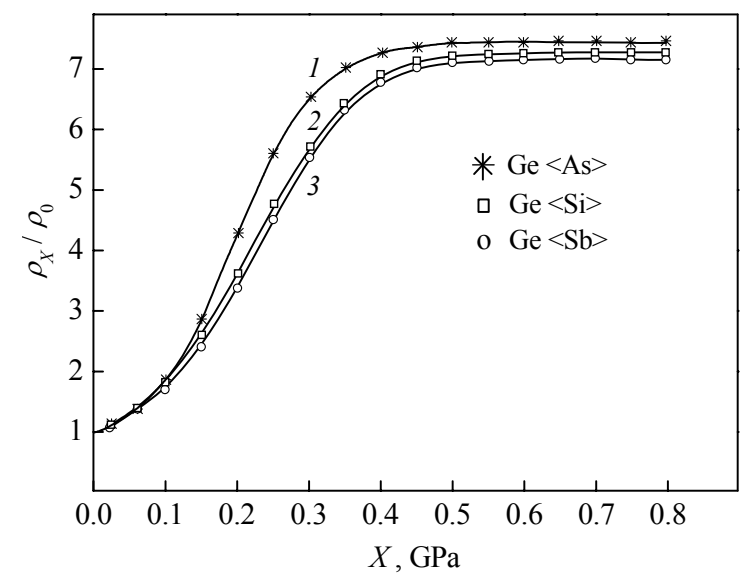

Fig. 1. Dependences of resistivity $\rho_{X} / \rho_{0}$ on the mechanical load $X$ under conditions $\vec{J}\|\vec{X}\|[111]$ obtained at $T=77.4 \mathrm{~K}$ in the experiments with $n$-Ge single crystals doped with various impurities: $1-\mathrm{As}, 2-\mathrm{Si}, 3-\mathrm{Sb}$. The resistivity of all the studied crystals is $\rho_{77.4 \mathrm{~K}} \approx 0.8 \ldots 0.9 \mathrm{Ohm} \cdot \mathrm{cm}$. 
Table. Electrophysical parameters of $n$-Si single crystals.

\begin{tabular}{|c|c|c|c|c|c|c|c|c|}
\hline \multirow{2}{*}{$\begin{array}{c}\text { Number } \\
\text { of sample }\end{array}$} & \multirow{2}{*}{$\begin{array}{c}\text { Impu- } \\
\text { rity }\end{array}$} & \multirow{2}{*}{$\begin{array}{c}\text { Tetrahedral } \\
\text { radius of } \\
\text { impurity, } \AA\end{array}$} & \multicolumn{2}{|c|}{ Resistivity $\rho$, Ohm·cm } & \multicolumn{2}{|c|}{$\frac{1}{R_{\mathrm{H}} e} \cdot 10^{-18}, \mathrm{~cm}^{-3}$} & \multicolumn{3}{|c|}{$\begin{array}{c}\text { Hall mobility } \mu_{\mathrm{H}}, \\
\mathrm{cm} / \mathrm{V} \cdot \mathrm{s}\end{array}$} \\
\cline { 5 - 10 } & & & $300 \mathrm{~K}$ & $4.2 \mathrm{~K}$ & $300 \mathrm{~K}$ & $4.2 \mathrm{~K}$ & $300 \mathrm{~K}$ & $4.2 \mathrm{~K}$ \\
\hline 1 & $\mathrm{P}$ & 1.10 & 0.0063 & 0.0030 & 8.0 & 8.80 & 124 & 237 \\
\hline 2 & $\mathrm{Sb}$ & 1.36 & 0.0066 & 0.0031 & 6.7 & 6.70 & 141 & 301 \\
\hline 3 & $\mathrm{As}$ & 0.96 & 0.0060 & 0.0072 & 9.0 & 9.36 & 116 & 93 \\
\hline
\end{tabular}

The values of the anisotropy parameter for mobility $K$, received from the tensoresistance experimental data and using the formula (1) for $\mathrm{Ge}\langle\mathrm{As}\rangle$ and $\mathrm{Ge}\langle\mathrm{Sb}\rangle$ crystals with practically the same level of doping, were slightly different to each other, namely: $K_{\mathrm{Ge}\langle\mathrm{As}\rangle}=10.65>K_{\mathrm{Ge}\langle\mathrm{Sb}\rangle}=10.23$. That is, the ratio of the anisotropy parameters of electron mobility in $\mathrm{Ge}$ samples doped with $\mathrm{As}$ and $\mathrm{Sb}$ is approximately only 1.04 , and hence does not exceed $4 \%$. First of all, it is caused by the predominant role of the scattering of charge carriers by vibrations of crystal lattice in the crystal of cubic system. The germanium crystals belong just to this cubic system.

It is characteristic that the use of impurity with higher tetrahedral radius $\left(r_{\mathrm{Sb}}=1.36 \AA>r_{\mathrm{Si}}=1.17 \AA>\right.$ $r_{\mathrm{As}}=0.96 \AA$ ) in Ge crystals is accompanied not only by a decrease of tensoresistance $\rho_{X} / \rho_{0}$ in the saturation region, but also by decrease in the rate of enhancing $\rho_{X} / \rho_{0}$ with increasing the pressure in the intermediate range of $X$ (between 0 and $X \rightarrow \infty$ ), which is manifested by a decrease in the slope of the curves 2 and 3 (Fig. 1).

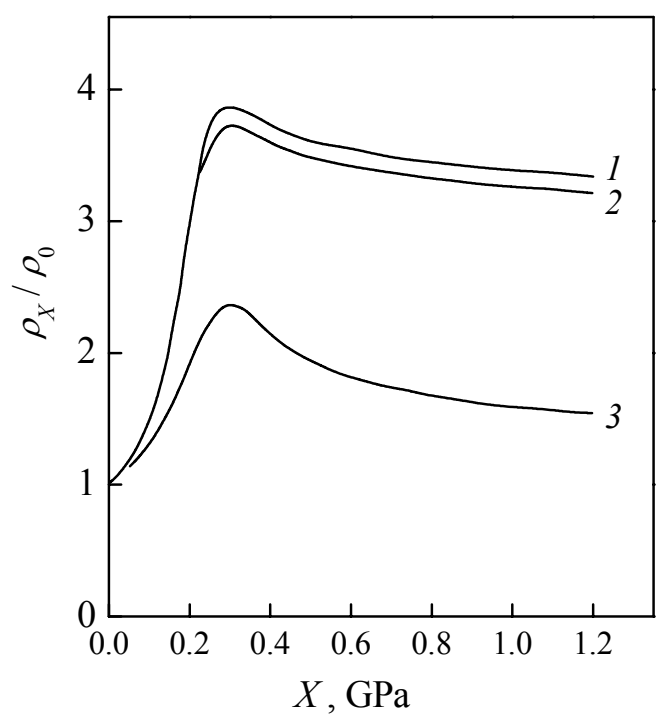

Fig. 2. Dependences $\rho_{X} / \rho_{0}=f(X)$ in $n$-Si under conditions $\vec{J}\|\vec{X}\|[001]$ and $T=4.2 \mathrm{~K}$. Curve numbers correspond to the sample numbers in Table.
This fact, in turn, describes the difference in the interaction of impurity centers with different tetrahedral radii, which is characteristic for the curves 1 to 3 (Fig. 1), between itself and with the crystal lattice.

Thus, if we compare the tetrahedral radii of used impurities $\mathrm{As}, \mathrm{Si}$ and $\mathrm{Sb}$ with those of the matrix atoms, then it is clear from Fig. 1 that the higher values of tensoresistance in saturation correspond to the smaller radii of impurities.

It was interesting to test whether the effect of impurities on tensoresistance in other many-valley material exists, meanwhile expanded the range of doping. In relation with it, dependencies of the longitudinal tensoresistance on mechanical strain $X$ were measured (Fig. 2) under conditions $\vec{J}\|\vec{X}\|[001]$ and $T=4.2 \mathrm{~K}$ in $n$-Si crystals heavily doped with phosphorus $\left(r_{\mathrm{P}}=1.10 \AA\right)$, arsenic As and antimony $\mathrm{Sb}$ (see parameters of the investigated samples in Table).

One can separate the following specific parts for each curve of tensoresistance $\rho_{X} / \rho_{0}$ in Fig. 2:

1) at the beginning, there is a weak dependence $\rho_{X} / \rho_{0}$ on the mechanical strain $X$;

2) the following strong growth of tensoresistance with an increase in $X$;

and finally,

3) region after maximum of dependence $\rho_{X} / \rho_{0}$, which only conventionally can be named "saturation", because there is a certain dependence of the resistivity $\rho$ on $X$ here.

The increase of the $\rho_{X} / \rho_{0}$ ratio is associated with redistribution of charge carriers in the region of the initial and low mechanical strain. This increase is accounted for a decrease in the electron mobility and is caused by two reasons.

First, two of six energy minima are dropped and four minima are raised under uniaxial compression of $n$-Si in the crystallographic direction $\langle 100\rangle$, and finally all the carriers (for some values of pressure $X$ ) are found in these two minima that are dropped. For the conditions $\vec{J}\|\vec{X}\|[001]$, the mobility of carriers that pass out of rising minima into descending minima decreases from the value determined by the transverse effective mass of the charge carriers $m_{\perp}$ to a value determined by the longitudinal effective mass $m_{\|}$. 


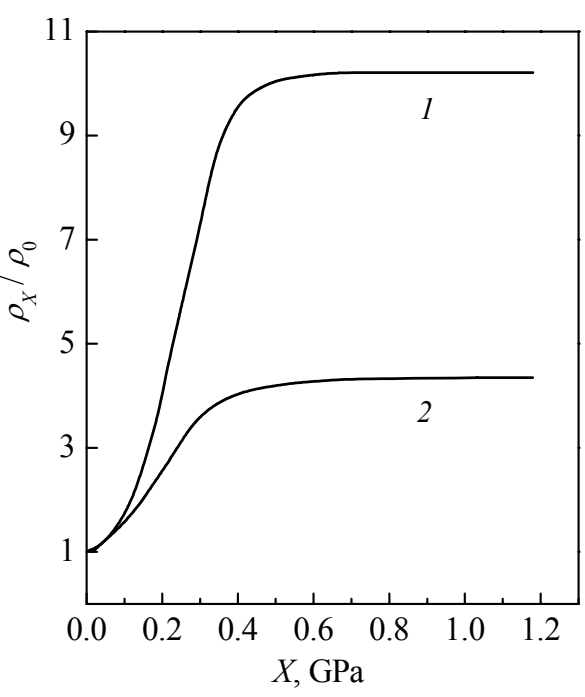

Fig. 3. Dependences $\rho_{X} / \rho_{0}=f(X)$ measured at $T=77.4 \mathrm{~K}$ in single crystals: $1-n-\mathrm{Ge}\langle\mathrm{Sb}\rangle, n_{e}=5 \cdot 10^{13} \mathrm{~cm}^{-3}, \vec{X}\|\vec{J}\|[111]$; $2-n-\mathrm{Si}\langle\mathrm{P}\rangle, n_{e}=7.1 \cdot 10^{13} \mathrm{~cm}^{-3}, \vec{X}\|\vec{J}\|[100]$.

Second, fluctuations in the concentration of the charged impurities that reduce the relaxation time $\tau$ and, consequently, the carrier mobility $\mu$, have an important impact on the scattering processes in highly doped silicon due to the increasing energy of electrons in these crystals with increasing the pressure $X$.

With further increase in $X$ (see Fig. 2) after reaching the maximum value, the tensoresistance $\rho_{X} / \rho_{0}$ begins to decline. Due to this fact, heavily doped crystals differ from relatively pure samples of silicon and germanium that have similar curves with the pronounced saturation (Fig. 3).

In germanium crystals without deep levels, the resistivity for the crystallographic direction [111] at the beginning increases with increasing the mechanical stress, and then reaches saturation (see Fig. 3, curve 1). The same feature is observed in silicon crystals when applying pressure in the direction [100] (Fig. 3, curve 2). The curves shown in Fig. 3 are typical for $n$-Ge and $n$-Si at $T=77.4 \mathrm{~K}$. It should be noted that the mentioned characteristic property (decline of the dependence $\rho_{X} / \rho_{0}=f(\mathrm{X})$ with increasing $\left.X\right)$ of heavily doped crystals occurs also in other experimental studies (see, for example, $[16,17])$.

With increasing the impurity concentration, the energy spectrum of charge carriers in heavily doped semiconductors significantly changes: in the forbidden band near the edges of the allowed bands the "tail" of the density of states appears - aggregate of the specific electronic states. Therefore, the decline of $\rho_{X} / \rho_{0}$ in the curves shown in Fig. 2 after reaching the maximum tensoresistance is advisable to link with the change of the energy position of the impurity states with respect to the bottom of the conduction band (C-band). The carrier concentration in the $\mathrm{C}$-band increases as the result of this.

It should be noted that the existence of the "tail" of state density in highly doped semiconductors, probably, does not only exclude, but even promotes occurrence of such deformation changes, since the deep impurity levels are weakly bound with the corresponding band (in particular, in $n$-Si with the bottom of C-band). Hereupon, the tensoresistance of these crystals is strongly dependent on the applied mechanical stress [18]. A similar effect can be expected in heavily doped semiconductors deformed in the crystallographic direction, which provides the removal of degeneracy of the energy bands, because in such semiconductors there are the electronic states located at the energy scale below the level formed by an isolated atom of the same impurity, i.e., rather deep in the forbidden band. In addition, these (deep) states in the density-of-state tails, as emphasized in [19], are more reminiscent of the impurity states at least because they are localized, i.e., the conductivity (mobility) on them is close to zero. Thus, the absence of saturation in the dependences $\rho_{X} / \rho_{0}=f(X)$ of heavily doped $n$-Si, is obviously related with the additional contribution to the conductivity of charge carriers of the impurity subsystem.

Taking into account all the above mentioned, it becomes clear why the drop of the dependence $\rho_{X} / \rho_{0}=f(X)$ for the samples doped with arsenic (Fig. 2, curve 3 ) is steeper as compared with the similar curves 1 and 2 (Fig. 2). The reason of this phenomenon is naturally associated with the individual physicochemical properties of the dopant impurity. One of them (important for the present case), probably, consists in the fact that the depth of the impurity levels of arsenic in silicon is greater than that of phosphorus, for example.

\section{Conclusions}

The analysis showed that the results of tensoresistance measurements in $\mathrm{Ge}$ and $\mathrm{Si}$ single crystals in a wide range of mechanical loads $X$ depend not only on the dopant concentration in the investigated crystal, but also on its physical and chemical identity. Even such a seemingly neutral parameter as the tetrahedral radius of the impurity atoms is manifested, as it turned out, in the form of the above-discussed features of dependencies $\rho_{X} / \rho_{0}=f(X)$ that are measured at all things being equal.

\section{References}

1. G.P. Gaidar, The Kinetics of Electronic Processes in Si and Ge in the Fields of External Influences: Monograph. LAP LAMBERT Academic Publishing, Saarbrücken, Deutschland, 2015 (in Russian).

2. O.V. Tretiak, V.V. Il'chenko, Physical Principles of Semiconductor Electronics. VPTs "Kyivskyi universytet”, Kyiv, 2011 (in Ukrainian).

3. Ya.V. Lepikh, Yu.O. Gordiienko, A.O. Druzhynin, Creating Microelectronic Sensors of New Generation for the Intellectual Systems: Monograph. Astroprynt, Odesa, 2010 (in Ukrainian). 
4. P.I. Baranskii, A.V. Fedosov, G.P. Gaidar, Heterogeneities of Semiconductors and Urgent Problems of the Interdefect Interaction in the Radiation Physics and Nanotechnology. Editorial and Publishing Department of the Lutsk State Technical University, Kyiv-Lutsk, 2007 (in Ukrainian).

5. P. Studer, S.R. Schofield, C.F. Hirjibehedin, N.J. Curson, Studying atomic scale structural and electronic properties of ion implanted silicon samples using cross-sectional scanning tunneling microscopy // Appl. Phys. Lett. 102 (1), p. 012107 (2013).

6. Defects in semiconductors / Ed. by A. Cavallini // Proc. of the 27th Internat. Conf. (ICDS-2013), 2126 July 2013, Bologna, Italy. Ser. AIP Conference Proceedings. American Institute of Physics (AIP), Curran Associates, Inc., Apr. 2014.

7. P. Studer, V. Brázdová, S.R. Schofield, D.R. Bowler, C.F. Hirjibehedin, N.J. Curson, Site-Dependent Ambipolar Charge States Induced by Group V Atoms in a Silicon Surface // ACS Nano 6 (12), p. 10456-10462 (2012).

8. P.I. Baranskii, A.E. Belyaev, G.P. Gaidar, V.P. Klad'ko, A.V. Kuchuk, Problems of Real Semiconductor Crystals Diagnostics. Naukova Dumka, Kiev, 2014 (in Ukrainian).

9. B.I. Shklovskii, A.L. Efros, Electronic Properties of Doped Semiconductors. Springer Science \& Business Media, 2013.

10. A.A. Grosa, P.G. Litovchenko, M.I. Starchyk, The Effects of Radiation in the Infrared Absorption and Structure of Silicon. Naukova dumka, Kyiv, 2006 (in Ukrainian).

11. G.P. Gaidar, Transformation of Radiation Defects and Kinetic Phenomena in Si and Ge: Monograph. LAP LAMBERT Academic Publishing, Saarbrücken, Deutschland, 2013 (in Russian).
12. V.M. Babich, N.I. Bletskan, E.F. Venger, Oxygen in Silicon Single Crystals. Interpress LTD, Kiev, 1997 (in Russian).

13. S.I. Budzuliak, Tensoresistive effects in strongly deformed $n$-Si and $n$-Ge crystals // Fizyka i khimiia tverdoho tila, 13(1), p. 34-39 (2012), in Ukrainian.

14. G.P. Gaidar, On the tensoresistance of $n$-Ge and $n$-Si crystals with radiation-induced defects // Fizika i tekhnika poluprovodnikov, 49 (9), p. 1164 1168 (2015), in Russian.

15. G.P. Gaidar, On methodology of measuring parameters with the increased sensitivity to residual or irradiation induced inhomogeneities in semiconductors // Semiconductor Physics, Quantum Electronics \& Optoelectronics, 12 (4), p. 324-327 (2009).

16. G.P. Gaidar, P.I. Baranskii, V.V. Kolomoets, Tensoresistance of $n$-Si and $n$-Ge multi-valley semiconductors over a wide range of concentrations // Fizyka i khimiia tverdoho tila, 15 (1), p. 58-62 (2014), in Ukrainian.

17. V. Kolomoets, V. Ermakov, L. Panasyuk, S. Fedosov, B. Orasgulyev, P. Nazarchuk, Application of high uniaxial strain methods for semiconductor parameter determination // Physica B: Condensed Matter, 417, p. 46-48 (2013).

18. S.A. Fedosov, S.V. Lunev, D.A. Zakharchuk, L.I. Panasyuk, Yu.V. Koval', The influence of uniaxial elastic deformation on the position and the degree of filling of the deep level $E_{C}-0.2 \mathrm{eV}$ in $n$-Ge $\langle\mathrm{Au}\rangle$ single crystals // Naukovyi Visnyk Volyns'koho Universytetu im. Lesi Ukrainki, Fizychni Nauky. No. 16, p. 39-45 (2011), in Ukrainian.

19. P.I. Baranskii, A.V. Fedosov, G.P. Gaidar, Physical Properties of Silicon and Germanium Crystals in the Fields of Effective External Influence. Nadstyr'e, Lutsk, 2000 (in Ukrainian). 